\title{
VARIA
}

\section{L'«UBAC» DES ÉTUDES DE RÉCEPTION DE LA TÉLÉVISION}

\author{
Brigitte Le Grignou ${ }^{1}$
}

Pour étudier d'un point de vue critique les promesses du mythe communicationnel (dont l'interactivité), E. Neveu ${ }^{2}$ forge le projet de montrer l'«ubac» du mythe. Nous lui empruntons cette métaphore pour dévoiler la face la plus sombre, la moins riante, des travaux sur la réception de la télévision.

Il ne s'agit pas d'une dénonciation de ces travaux au nom d'une ligne "politiquement correcte" mais d'un repérage des écueils et des impasses, bref, de l'affirmation d'une nécessaire vigilance pour qu'ils restent féconds.

Les études de réception et les réactions qu'elles suscitent sont révélatrices d'un débat qui, comme le note justement D. Pasquier ${ }^{3}$, "dépasse largement le cadre d'une opposition entre partisans d'une théorie des médias puissants et partisans d'une théorie des téléspectateurs puissants. Ce qui est ici au cœur du problème c'est la question du statut social qu'il faut donner au média. Et c'est sans doute la question que n'a cessé de poser la sociologie de la

\footnotetext{
Maître de conférences à l'Université Paris-Dauphine

2 E. Neveu, Une société de communication?, Paris, Montchrestien, 1994.

3 D. PASQUIER, "Vingt ans de recherches sur la télévision", Sociologie du travail, $n^{\circ} 4,1993$.
} 
télévision". Cette question de l'inscription de la télévision dans le débat social, qu'elle soit posée en termes de lien social, d'espace public, de contrôle social ou de société civile, peut donner lieu à des "dérives mythologiques"1. Trois types en sont repérables dans les travaux sur la réception:

1. Le mirage du public ${ }^{2}$ qui confère au groupe des téléspectateurs les apparences séduisantes et trompeuses d'une communauté.

2. La dérive populiste qui tend à substituer aux victimes aliénées des médias de masse des acteurs alertes et "activement employés, face à leur poste, à épingler les connotations furtives, à résister aux séductions hégémoniques, et en général, à déjouer les ruses de l'idéologie"3.

3. L'illusion optimiste qui, affichant une confiance résolue dans les vertus pédagogiques et émancipatrices de la télévision, évacue la problématique de pouvoir.

\section{Le mirage du public}

D. Dayan, à propos des cérémonies retransmises par la télévision, remarque que le médium doit faire face à un problème qui a longtemps hanté, entre autres, la liturgie juive: "Comment créer une communauté de célébration lorsque les membres de la collectivité concernée sont physiquement séparés?"4. Si la question est particulièrement fondée en ce qui concerne les cérémonies télévisées, elle nous paraît applicable à tout genre télévisuel dont la réception est essentiellement "diasporique".

Cette séparation n'est certes pas une spécificité des téléspectateurs, elle caractérise aussi les lecteurs de la presse et plus généralement les usagers de technologies d'information et communication (dont la "consommation" est le plus souvent individuelle et s'effectue dans la sphère domestique ou plutôt privée). C. Chabrol ${ }^{5}$ rappelle

I D. DAYAN, "Les mystères de la réception", Le Débat, nº 74, 1992.

2 P. SORLIN, "Le mirage du public", Revue d'histoire moderne et contemporaine, $\mathrm{n}^{\circ} 39,1992$.

3 D. MORLEY, "La «réception» des travaux sur la réception", Hermès, n 11/12, 1993.

4 D. DAYAN et E. KATZ, "Rituels publics à usage privé", Annales, n 1, 1983.

5 C. Chabrol, "Le lecteur: fantôme ou réalité?", in P. Charadeau (dir.), La presse, produit, production, réception, Paris, Didier Érudition, 1988. 
opportunément la particularité des discours médiatiques: ils sont "monologiques" et donc "le cadre situationnel pose une interaction médiate et indéterminée sans rétroaction interlocutive". Cette construction monologique ne repose que sur une série de paris psycho-socio-sémiotiques. Ce sont des communications "imaginaires" et les paris sont d'autant plus hasardeux que le producteur ne peut viser que des destinataires collectifs ou génériques (cibles, publics). Bien sûr, on peut trouver le lecteur dans la fiction (Lector in fabula), un lecteur-spectateur "épistémique" est saisissable au niveau de ce qui dans le texte est conçu pour lui. Mais qu'en est-il du spectateur "empirique" qui interprète le texte sans percevoir les indices qui lui étaient destinés? Où trouver la preuve d'une coïncidence harmonieuse, providentielle, entre les destinataires idéaux construits par le discours médiatique et les destinataires réels?

Le développement de la communication de masse a suscité les réflexions de philosophes, sociologues ou historiens sur la notion de public. Nombre de définitions n'autorisent pas à constituer les téléspectateurs en public. On sait ainsi que pour J. Habermas, la consommation de culture (et il cite notamment en exemple le fait de regarder ensemble la télévision) est une activité de groupe qui ne suscite aucun public. Car, dans le passage de la culture discutée à la culture consommée, "rien ne subsiste des relations caractéristiques d'une sphère privée corrélative d'un public"1. Certes des discussions ont lieu, mais elles sont administrées et revêtent une forme marchande: 'L'usage que le public des lecteurs faisait de sa raison tend à s'effacer au profit de simples opinions sur le goût et l'attirance qu'échangent des consommateurs"2.

On peut certes trouver chez G. Tarde, à propos du lectorat de la presse écrite à la fin du siècle dernier, une définition moins restrictive et transposable aux téléspectateurs. Il envisage en effet le public comme "une collectivité purement spirituelle, comme une dissémination d'individus physiquement séparés et dont la cohésion est toute mentale"3. Mais il précise cependant que la constitution d'un public de journal suppose "l'intérêt pour les affaires publiques": "les lecteurs, même habituels d'un journal, tant qu'ils ne lisent que les

1 J. Habermas, L'espace public, Paris, Payot, 1978, p. 171.

2 Ibidem, p. 179. J. Habermas note également: "Ainsi transformé, l'usage de la raison remplit certes d'importantes fonctions psychosociologiques; il est avant tout un substitut rassurant de l'action" (p. 172).

3 G. TARDE, L'opinion et la foule, Paris, Alcan, 1901, p. 2. 
annonces et les informations pratiques se rapportant à leurs affaires privées, ne forment pas un public"'. Y. Lavoine ${ }^{2}$ voit dans ce critère l'émergence d'un public "idéologique", spécifique à tel journal particulier et, en tant que tel, étranger au "grand public": "C'est du moment où les lecteurs d'une même feuille se laissent gagner par l'idée ou par la passion qui l'a suscitée qu'ils composent vraiment un public"3.

Pour P. Sorlin ${ }^{4}$ qui se penche en historien sur la conception du public comme entité sociale, un public est "une communauté passagère qui, cependant, a ses règles et ses rites et qui ne se dissout pas quand l'occasion de son rassemblement est passée". Le facteur externe, c'est-à-dire l'occasion qui rapproche fugitivement, ne suffit pas, on le voit, à constituer un public. Il faut aussi que des modes de participation au groupe dont on fait temporairement partie, la fixation d'objectifs, la recherche de moyens propres à rejoindre le but poursuivi le cimentent durablement.

On peut bien sûr définir le public comme une "communauté imaginée" (selon la définition que $\mathrm{B}$. Anderson ${ }^{5}$ applique à la nation). Mais le sentiment d'appartenance à la nation s'affirme, s'incarne, s' "objective" dans une langue, des cérémonies, des rituels. En ce qui concerne le public de télévision, ces manifestations sont exceptionnelles. On en trouve des traces par exemple lorsque les téléspectateurs se réunissent en associations ou lors de cérémonies télévisées ${ }^{6}$, mais ces expressions d'appartenance à une communauté qui "ne se dissout pas quand l'occasion de son rassemblement est passée" demeurent très ponctuelles. On pourrait enfin essayer de cerner un ou des publics spécifiques, par exemple les "petits" consommateurs de télévision. Mais l'étude de leur consommation par genres ne révèle pas de spécificité par rapport aux "gros" consommateurs, ce qui signifie, comme l'a montré M. Souchon 7 qu'il n'y a pas, dans le cadre de chaînes généralistes, de publics spécifiques pour des émissions particulières. Le public des émissions à petit public n'est pas minoritaire, singulier,

1 Ibidem, p. 52.

2 Y. LAvoINe, "Le quotidien ou le collège invisible", in Actes du IXème congrès national des Sciences de l'information et de la communication, Toulouse, 1994.

3 G. TARDE, op. cit., p. 52.

4 P. SORLIN, art. cité.

5 B. ANDERSON, Imagined communities, London, Verso, 1991.

6 D. DAYAN et E. KATZ, art. cité.

7 M. Souchon, "Les programmateurs et leurs représentations du public", Réseaux, $\mathrm{n}^{\circ} 39,1990$. 
"éclairé", mais est constitué pour l'essentiel par ceux qui regardent beaucoup la télévision. La perspective d'A. de Tocqueville', en quête d'un antidote à l'individualisme, permet en revanche sinon de définir du moins de supposer un public. Il envisage, en effet, le journal (qui conjugue deux impératifs communicationnels: l'identité du message et la simultanéité de la réception) comme un vecteur de lien social. Le journal figure donc une communauté invisible à laquelle il fournit "un moyen de se parler tous les jours sans se voir, et de marcher d'accord sans être réunis" 2 . Cette conception "volontariste" du lectorat peut être mise en parallèle avec celle de D. Wolton relative aux téléspectateurs: "Le spectateur, en regardant la télévision, s'agrège à ce public potentiellement immense et anonyme qui la regarde simultanément, et entretient, de ce fait, avec lui une sorte de lien invisible"3. Certes, le lien invisible, silencieux, fait du partage de références communes est aussi qualifié de "ténu", mais il semble néanmoins exister sans que l'on sache précisément quelle est la nature du lien qui unit un ensemble de personnes dans l'identité apparente d'une pratique. Certes, cet ensemble englobe des individus qui présentent des caractéristiques communes dont la sociologie peut rendre compte: la consommation de télévision se distribue par exemple selon l'âge. Mais le trait dominant des lecteurs de journaux ou spectateurs demeure l'atomisation, et le chercheur, faute de cerner une identité d'intérêt ou un sentiment identitaire, est contraint de le postuler. Le problème devient alors celui de ce sentiment d'appartenance à une communauté et surtout de l'expression de ce sentiment.

L'atomisation, le faible degré d'organisation et d'expression des usagers de la télévision les rapprochent d'un "groupe latent", au sens que $\mathrm{R}$. Boudon donne à ce terme (c'est-à-dire un ensemble d'individus qui ont un intérêt commun mais qui, inorganisés, ne font rien pour promouvoir cet intérêt) ${ }^{4}$. L'expression des intérêts, des demandes de ce groupe, sa représentation, sont assurées par des "entrepreneurs" extérieurs, porte-parole qui diffusent le savoir sur cette communauté. C. Meadel ${ }^{5}$ voit par exemple dans les premiers sondages organisés par

1 A. DE Tocqueville, De la démocratie en Amérique (t. 2, 1840), Paris, GarnierFlammarion, 1981.

2 Y. LAVOINE, op. cit., p. 174.

3 D. Wolton, Eloge du grand public, Paris, Flammarion, 1990, p. 126.

4 R. Boudon, Préface à M. Olson, Logique de l'action collective, Paris, P.U.F., 1978.

5 C. MeAdEL, "De la formation des comportements et des goûts", Réseaux, $n^{\circ} 39$, 1990. 
la télévision, dans les années cinquante, un mode de constitution, d' "invention" du public. Ces sondages consistent d'abord à parler de la télévision, à la faire connaître (il n'est nulle part indiqué que le sondage puisse changer quoi que ce soit dans le contenu des programmes). Ils ont ensuite pour objectif de faire comprendre au spectateur ce qu'il doit faire avec la télévision et la place qu'il doit occuper. Cette place est celle d'un "sujet de goût" qui doit apprécier, évaluer; on lui fournit alors les catégories, les modes d'approche, les questions qu'il doit se poser et les types de réactions légitimes.

Il est une seconde dimension importante et spécifique du rapport à la télévision qui ne facilite pas la saisie du public. La télévision souffre, comme le notent P. Chambat et A. Ehrenberg1, d'un "déficit de légitimité" tel que, quelles que soient les pratiques, le discours tenu est celui d'une mise à distance: "On n'est pas tellement télé" 2 . Devant le risque de dilution dans la masse des téléspectateurs, chacun s'efforce de mettre en œuvre des stratégies de résistance à l'inclusion, de souligner son particularisme pour briser l'unité fictive de cette population, définie de façon quasi technique (locataires d'un immeuble, usagers de la SNCF, possesseurs de téléviseurs). Donc si sentiment d'appartenance il y a, c'est un sentiment honteux, rarement revendiqué, le plus souvent dénié. L'argument est certes insuffisant pour nier l'existence d'une communauté mais suffisant pour pointer la singularité de ce groupe social "imaginé" par rapport à des homologues comme la nation ou même le lectorat de la presse.

Cet acharnement distinctif des téléspectateurs non seulement désigne mais aussi récuse une opération simplificatrice: le cantonnement des membres du public à un seul univers d'appartenance. Or, pour être téléspectateur, cesse-t-on pour autant d'être femme, amateur de jeux de boules ou de tennis, catholique pratiquant, communiste, ou membre de groupes aussi informels que "les myopes, les grands nez, les anciens du Club, les échangistes, les allergiques au pollen"3.

De cette difficulté à envisager la pluralité d'appartenance des individus à des "publics" divers, témoignent certaines recherches menées dans une perspective ethnographique et notamment les travaux de J. Lull sur les usages de la télévision dans le cadre fami-

\footnotetext{
1 P. Chambat et A. Ehrenberg, "De la télévision à la culture de l'écran", Le Débat, $n^{\circ} 52,1988$.

2 D. BouLLIER, La conversation-télé, Rennes, Lares, 1987.

3 Ibidem.
} 
lial ${ }^{1}$. L'auteur qui recueille les données par observation participante, fait preuve d'une réelle prudence et rigueur méthodologiques. Mais ses conclusions souffrent d'une réduction à l'univers familial, à ses pratiques et à ses rôles. Est ainsi élaborée une typologie des usages sociaux de la télévision, qui distingue "usages structurels" (par exemple, la télévision est considérée comme "régulateur de comportement", qui ponctue le temps et les activités de la famille et de ses membres) et "usages relationnels". Ceux-ci sont de quatre types: "Communication facilitator", les programmes ou le fait même de regarder génèrent des conversations, procurent histoires ou thèmes ("agenda for talk"). "Affiliation/Avoidance": la télévision est alors une ressource pour la construction d'opportunités de contacts interpersonnels ou d'évitements. "Competence/Dominance": la télévision est utilisée pour démontrer une compétence (par exemple linguistique) et/ou une suprématie sur les autres membres de la famille. "Social learning": l'acquisition de savoirs se fait alors non seulement par les informations mais aussi par les feuilletons qui procurent des modèles pour les interactions sociales, des modèles de rôles. Seul ce dernier type de ressource procurée par la télévision ouvre l'univers domestique au monde social, mais les médiations entre les deux (modes d'accès aux programmes et construction des jugements, modes d'acquisition des téléviseurs ou autres "objets domestico-médiatiques"2) sont ignorées et demeurent une énigmatique "boîte noire".

\section{La dérive populiste}

La dérive populiste aboutit tantôt à un portrait du téléspectateur en libre consommateur, tantôt à l'image d'un héros qui résiste vaillamment aux séductions hégémoniques.

\section{Le consommateur libre}

Certes, les études de réception ne proposent pas une activité du spectateur détachée de tout contexte. Celle-ci est par exemple enca-

1 J. LuLL, Inside Family Viewing, London-New York, Routledge, 1990.

2 G. DERÈZE, Une ethnosociologie des objets domestico-médiatiques, Louvain-laNeuve, CIACO, 1994. 
drée dans une communauté culturelle qui lui fournit une grille de lecture et des ressources interprétatives ${ }^{1}$ ou dans un contrat spécifique au genre télévisuel consommé ${ }^{2}$. Mais ces données contextuelles apparaissent parfois très proches de catégories psychologiques. Ainsi, J. Bianchi analyse-t-il la notion de genre (forme de contrat implicite entre les parties prenantes de la situation de communication) dans son rapport aux pratiques de réception. Il propose une typologie des logiques de réception déterminées par les divers genres télévisuels: il distingue une "logique joueuse", manière spécifique de participer aux jeux, forme particulière de comportement et de gratification, une "logique de la fiction", manière de se positionner dans un récit et de "retoucher" son rapport au monde, une logique des "current affairs" qui satisfait la curiosité, le besoin de repères sur l'environnement et les événements, et une "logique du spectacle", qui relève du plaisir de ce qui est donné à voir. Ces logiques, construites sur les "gratifications" en vue de reconstituer les besoins auxquels elles correspondent, ne sont pas sans évoquer la typologie des "usages et gratifications" et la notion de besoin qui la fonde (cognitive needs, affective needs, integrative needs, contact, escapism). Comment ne pas voir, à la suite de P. Beaud, dans cette référence aux "besoins", une variation sur la "nature de la demande", qui ne s'interroge jamais sur la position de la demande dans la structure de la formation sociale. Comment ne pas s'interroger sur les "significations sociales du fait que le rapport des spectateurs aux mass-médias (...) soit vécu par ce spectateur comme un besoin «librement» et «spontanément» éprouvé"3. L'individu paraît dialoguer avec les médias en fonction de ses besoins qui préexistent à ceux-ci, l'audience est considérée comme active, c'est-à-dire que l'usage qu'elle fait des médias est orientée vers un but, le médium remplit alors une fonction. On oublie ou on feint d'oublier que l'émetteur est toujours à la source et que, comme dans un marché, il n'existe pas une demande préalable qui se construirait sur des besoins "naturels".

De plus, l'analyse en termes de besoins conduit à négliger la capacité inégale des spectateurs à interpréter et à verbaliser leur rapport à leur pratique, ou "l'inégale distribution sociale des

1 T. LIEBEs, “A propos de la participation du téléspectateur”, Réseaux, n ${ }^{\circ}$ 64, 1994.

2 J. BIANCHI, "Les genres dans la pratique de la réception télévisuelle", in C. BELISLE (dir.), Communication et nouvelles technologies, Lyon, Programme Rhône-Alpes de Recherches en Sciences humaines, 1993.

3 P. BEaud, La société de connivence, Paris, Aubier-Montaigne, 1984, p. 121. 
ressources discursives". "Les publics ne disposent pas d'un répertoire infini de discours interprétatifs, face aux significations proposées par la TV. La place des individus dans la structure sociale tend à déterminer la nature des discours qui leur sont aisément accessibles. La disponibilité de ces discours influencera l'éventail d'interprétations qu'ils offriront du contenu des médias"1.

Cette valorisation de l'autonomie du récepteur, liée à une sousestimation des logiques industrielles et sociales qui construisent le rôle du téléspectateur, apparaît encore plus nettement à l'œuvre dans le travail de J. Fiske sur la culture populaire ${ }^{2}$. Il s'agit pour cet auteur de mettre en lumière l'autonomie (relative?) des agents dans la production de leur culture populaire: "La culture populaire est faite par les gens, et non pas produite par les industries culturelles. Tout ce que ces industries peuvent faire est de produire un répertoire de textes ou de ressources culturelles que les diverses formations sociales utiliseront ou rejetteront, dans un processus permanent de production de leur culture populaire"3. On a la vision d'un spectateur-consommateur qui fait son marché avec une claire conscience des différents produits offerts et de ce qu'il peut en attendre. La rationalité des médias est alors réduite à une fonction sociale de satisfaction des besoins individuels et collectifs.

Peut-être convient-il, aujourd'hui, de penser les médias, même et surtout dans les études de réception, dans les termes que J. K. Galbraith appliquait à la production industrielle: "l'homme n'est un objet de science pour l'homme que depuis que les automobiles sont devenues plus difficiles à vendre qu'à fabriquer". Le problème des médias résidant moins dans la production de programmes que dans son écoulement, il s'agirait donc de "produire" des consommateurs.

\section{Le téléspectateur héroïque}

Les thèmes de l" "appropriation" du message télévisuel et de la "résistance" sont mis en avant comme des signes de ralliement à un

1 J. Curran, "La décennie des révisions", Hermès, n 11/12, 1993.

2 J. Fiske, Understanding Popular Culture, Boston, Unwin Hyman, 1989.

3 Ibidem, p. 24: "Popular culture is made by the people, not produced by the culture industry. All the culture industries can do is produce a repertoire of texts or cultural resources for the various formations of the people to use or reject in the ongoing process of producing their popular culture". 
public actif, critique et libre. La référence majeure de nombre de travaux est M. de Certeau'. Or, si celui-ci dédie son livre à "l'homme ordinaire", "héros anonyme" qui invente le quotidien, il n'abandonne jamais sa lucidité sur la force (ou la faiblesse) réelle de ce héros. Pour lui, le registre des usagers est celui de la tactique et non de la stratégie. La tactique est ce qui reste aux dominés, le dernier recours des faibles, des non-producteurs de la culture, qui tentent de détourner l'ordre des choses "sans illusion qu'il va changer de sitôt": "La tactique n'a pour lieu que celui de l'autre. Aussi doit-elle jouer avec le terrain qui lui est imposé tel que l'organise la loi d'une force étrangère. (...) Elle est mouvement à l'intérieur du champ de vision de l'ennemi, et dans l'espace contrôlé par lui'"2. Or, comme le note justement D. Dayan ${ }^{3}$, cette dimension de la réflexion de M. de Certeau tend à être occultée et "de dérisoire, ce bricolage devient triomphal".

J. Fiske fournit de cette dérive une remarquable illustration. Il analyse les réactions de jeunes aborigènes d'Australie à des westerns américains traditionnels, diffusés à la télévision. Il note que les téléspectateurs s'identifient aux Indiens, s'allient à eux et les encouragent lors des attaques de trains ou de fermes. Certes, précise-t-il, la défaite des Indiens est inévitable à la fin du film, mais cette défaite, loin d'imposer un sens univoque au message, est présentée comme une condition essentielle de son possible détournement: "Le plaisir procuré par la victoire des Indiens au milieu du film est, en partie, dépendant de leur inévitable défaite à la fin"4. De même, trouve-t-il dans la lettre d'une spectatrice assidue d'une émission de divertissement ("Newly Wed Game"), et dans le plaisir qu'elle dit éprouver, la marque d'une attitude subversive face à l'idéologie patriarcale ${ }^{5}$. On repère ici la substitution, évoquée par J. Curran, du plaisir ou de

1 M. DE CERTEAU, L'invention du quotidien. I, Arts de faire, Paris, Gallimard, coll. Folio, 1990 (1e édit. 1980).

2 Ibidem, p. 60. Il convient toutefois de signaler que pour C. Grignon, la démarche de M. de Certeau, lui-même, n'échappe pas à cette réduction "populiste". L'auteur y voit "une bonne illustration du magistère que les intellectuels (...) prétendent exercer, plus ou moins consciemment, sur les cultures dominées, et qui en fait un protectorat: cela consiste à décider, à la place des indigènes, de ce qui, dans leur culture, est beau, remarquable, intéressant, bref de ce qui a valeur, et qu'on entend faire voir et réhabiliter...". "Entretien avec C. Grignon", Politix, n 13, 1991.

3 D. DAYAN, art. cité.

4 J. FISKE, op. cit., p. 25: "The pleasure in the Indians' successes in the middle of the Western narrative is, in part, dependant on their inevitable defeat at the end".

5 IDEM, "Ethnosemiotics: Some personal and theorical Reflections", Cultural Studies, vol. $4, n^{\circ} 1,1990$. 
l'esthétique populaire au souci du politique: "On ne se demande plus si les représentation des médias font avancer ou reculer les luttes, on se demande d'où vient le succès des médias de masse" 1 . De plus, sauf à prêter à l'ensemble des spectateurs une posture de critique radicale, on peut s'interroger sur l'efficacité de cette subversion. Car si les femmes subvertissent un texte sexiste, que font les hommes du même texte? Si les jeunes aborigènes australiens subvertissent un message affirmant la supériorité de la race blanche, que font du même message les jeunes Américains blancs?

La construction d'un spectateur hérö̈que est fondée sur la négation des contraintes du texte et des genres. Ainsi, la pluralité des codes à l'œuvre dans un texte (polysémie généralisée) autoriserait à percevoir tous les textes comme également ouverts et susceptibles d'interprétations. Or, il est rare que les textes des médias soient complètement "ouverts" aux lectures. Leur niveau dénotatif guide, de façon plus ou moins marquée (variable selon les genres), la compréhension du public. Les textes comportent ce que D. Morley ${ }^{2}$ appelle des "éléments directifs de clôture du sens" (titres, énoncés autorisés), une "polysémie structurée". Sans faire du petit écran une "machine démoniaque qui manipule les spectateurs comme des marionnettes", il faut garder présent à l'esprit que "la télévision est l'incarnation d'un système de contraintes causales, d'un réseau de frontières qui limitent l'éventail des informations, des images et des représentations disponibles"3. La liberté du téléspectateur est donc conditionnelle et restreinte et la prolifération des lectures ne saurait remédier à ces circonstances.

\section{L'illusion optimiste}

L'illusion optimiste nourrit l'espoir d'une télévision génératrice de lien social, voire pour certains, constitutive d'un nouvel espace public.

Pour J. Bianchi et $\mathrm{H}$. Bourgeois, l'enjeu des études de réception est clairement pédagogique, il s'agit d'initier le spectateur à une

1 J. CuRran, "La décennie des révisions", art. cité, p. 56.

2 D. MORLEY, "La «réception» des travaux sur la réception", art. cité, p. 35.

3 W. Seaman, "La théorie du public actif: un populisme non fondé, Dossiers de l'audiovisuel, Paris, INA, n 51, 1993. 
réception lucide et active. Les deux auteurs présentent leur ouvrage comme "une invite à chacun de se faire récepteur plus qualifié, plus accompli"'. Ce projet ambitieux semble toutefois constamment se heurter à des limites dont les auteurs manifestent une claire conscience, sans aller jusqu'à les désigner. Le propos reflète comme une hésitation entre une vision enchantée d'une "culture de la réception" et un regard lucide sur les conditions de cette réception: "Rien n'empêche que nous puissions tenir notre place dans une partie qui a été distribuée sans nous mais dont nous ne sommes pas dupes"2. Ou encore: "La résistance qu'inclut la réception est donc en fait plus importante que ce que l'on croit parfois quand bien même elle ne va pas toujours assez loin dans la mise en ouvre de ses possibilités"3. Cette pédagogie de la réception poursuit un double objectif: il s'agit d'une part de développer le potentiel de résistance des usagers des médias: "Celle-ci [la réception] a beau être quelque peu prévisible, elle n'est jamais strictement nécessaire et elle est habituellement en mesure de faire valoir sa relative liberté. Elle a capacité à négocier, elle exerce à ce titre un certain contre-pouvoir ${ }^{4}$. Nous ne voulons pas majorer cette capacité (...). Une culture de la réception doit la développer"s. Il s'agit d'autre part de permettre une mobilisation du public comme groupe social. On retrouve ici, à travers une référence explicite aux travaux de D. Wolton, la thématique du lien social (certes "limité et fragile") généré par les médias, et des téléspectateurs unis par un sentiment d'appartenance à une communauté: "Les uns et les autres réalisent, au moins confusément, qu'ils constituent un public et que ce public a des réactions assez souvent communes, ce qui lui donne une réelle capacité d'influencer ce qui lui est destiné6. Les pas sont franchis de "l'appropriation" à la "résistance", mais surtout de la résistance à la mobilisation active.

Le travail de S. Livingstone et P. Lunt ${ }^{7}$ sur les débats avec participation du public franchit une nouvelle étape dans la célébration de la fonction émancipatrice de la télévision. Attentifs à la diversité des genres télévisuels, les auteurs analysent les émissions de discussion

1 J. Bianchi et H. Bourgeors, Les médias côté public, Paris, Centurion, 1992, p. 43.

2 Ibidem, p. 59.

3 Ibidem, p. 67.

4 Souligné par nous.

5 Ibidem, p. 69.

6 Ibidem, p. 79. Souligné par nous.

7 S. LivingSTONE et P. LuNT, "Se faire entendre dans l'espace public. Les femmes, la télévision et le citoyen téléspectateur", Réseaux, n 63, 1994. 
avec le public comme un genre nouveau et hybride qui bouleverse les frontières traditionnelles de l'information et du divertissement. Ces émissions (anglaises et américaines) leur paraissent susceptibles de servir de "forum féminin" dans l'espace public des médias: "Elles ouvrent un espace pour les individus ordinaires -sous la forme d'un vaste public de studio- qui autorise la rencontre et la discussion, sans délégation de paroles, à propos de leurs expériences personnelles, et en accord avec leur propre hiérarchie d'intérêt"'. L'analyse repose sur une critique de l'engagement et de l'action politiques traditionnels, perçus comme élitistes (dans le recours aux experts) et sexués ("le citoyen a toujours été conçu comme masculin"). Dans cette optique, les mass-médias ont un rôle particulier à jouer, et notamment les discussions télévisuelles entre gens ordinaires qui peuvent représenter une ressource pour une nouvelle sorte d'espace public, à condition de faire du politique "non plus la confiance en des programmes politiques formels mais en la circulation d'énoncés discursifs. En ce sens, le talk show peut être vu comme un terrain de lutte entre les pratiques discursives"2. La volonté des auteurs de célébrer le nouveau rôle de la télévision et corrélativement de dénoncer le "préjugé universitaire anti-télévision" est telle, qu'à l'appui de leur thèse, ils citent une déclaration, en forme de profession de foi, de la productrice et animatrice d'une de ces émissions (Oprah Winfrey): "Nous faisons ces émissions pour donner du pouvoir aux femmes"3.

Notre propos n'est pas de dénoncer le cynisme ou la naïveté des acteurs justifiant leur propre pratique mais de pointer la dénégation des logiques de pouvoir qui travaillent, dans ces débats, à imposer les problématiques, les registres d'intervention, à définir les situations de communication. On est ainsi en droit de se demander quels sont précisément les processus et questions politiques injectés dans l'espace domestique. En d'autres termes, de quoi parle-t-on à la télévision quand on parle de politique? Un début de réponse nous est proposé quand les auteurs évoquent "l'accès direct à des questions politiques que toutes les femmes peuvent trouver psychologiquement plus abordables" ou quand ils affirment que regarder à la télévision un débat entre député et citoyens est un acte politique. S. Livingstone et $\mathrm{P}$. Lunt font certes allusion à l'existence de "sévères restrictions au

\footnotetext{
1 Ibidem, p. 63.

2 Ibidem, p. 67.

3 Ibidem.
} 
nombre de discours possibles à l'intérieur de ce forum"', mais cellesci ne semblent pas les décourager tant est ancrée leur foi en une télévision, instrument de lutte politique. Cette vision résolument optimiste leur interdit de s'interroger davantage sur les conditions de conversion de ces formes de participation individuelle à un engagement collectif dans une action politique. Ils s'en remettent, eux aussi, à la magie d'un sentiment d'appartenance à une communauté: "Si nous pouvons tous nous considérer comme réunis par les massmédias en tant qu'imaginaire ou communauté symbolique ${ }^{2}$, partageant des significations et une connaissance auxquelles seuls les média nous donnent accès, alors la participation active -par la présence sur un plateau de télévision ou simplement chez soi devant sa télévisionpeut représenter une mutation du mécanisme de formation de l'opinion, de l'engagement et de l'action politique"3.

D'autres auteurs portent sur un genre proche (le talk show), un jugement radicalement différent. Ainsi, G. Lochard et J.-C. Soulages n'y voient qu'un "dérisoire artifice de compensation" pour l'exercice démocratique ${ }^{4}$. P. Chambat reconnaît que la télévision peut être un moyen de faire accéder à la visibilité des "mobilisations sociales plus ou moins éphémères et des revendications de justice éclatées" mais ne perçoit là que les facettes d'un "civisme sans citoyenneté, d'une solidarité (compassion) qui ferait l'économie d'un engagement politique"5. E. Macé, enfin, dans une étude intitulée La télévision du pauvre montre clairement comment la consommation/participation aux jeux télévisés est le fait de populations d'autant plus consommatrices de télévision qu'elles sont socialement non participantes et donc que regarder ou participer est une forme substitutive de participation sociale: "Le succès de la télévision populaire se fonde sur une double rupture: rupture du lien social et rupture entre le social et le politique. C'est cette double rupture que la télévision offre de compenser auprès de populations qui n'ont pas d'alternatives immédiates. De la sorte, en situation de monopole, l'industrie culturelle qu'est la télévision impose sa propre définition de la sociabilité et de la participation"6.

1 Ibidem, p. 68.

2 B. ANDERSON, op. cit.

3 S. LiVINGSTONE et P. LUNT, op. cit., p. 66.

4 J.-C. Soulages et G. LOChARD, "Les imaginaires de la parole télévisuelle", Réseaux, $\mathrm{n}^{\circ}$ 63, 1994.

5 P. Chambat, "Télévision et culture politique", XXème Siècle, nº 44, 1994.

6 E. MACE, "La télévision du pauvre", Hermès, nº 11/12, 1993, p. 172. 
D. Boullier, analysant les jugements des téléspectateurs sur la télévision, distingue trois types d'argumentations: la pratique ("je regarde"), le goût ("j'aime"), le jugement ("c'est bien"). Il note que cette clôture des arguments référés au seul univers télévisuel, si elle réalise au mieux le statut de téléspectateur entièrement pris dans l'univers du médium, inquiète pourtant les programmateurs et les annonceurs "qui sont toujours persuadés qu'il doit bien exister une «raison» aux comportements des téléspectateurs, sous-entendu une raison non télévisuelle, une raison «en béton» sur laquelle il serait possible de faire se combiner des variables, de découper des segments de population"'.

Chaque professionnel, afin de donner aux choix des usagers une rationalité ultime, va se référer à une cause extérieure à la télévision, les contraintes du milieu, les appartenances socio-culturelles ou les valeurs dominantes. Mais, note D. Boullier, "rien ne prouve que cette mise en cause possède un quelconque fondement (...). Cette opération ne marche que grâce à la capacité de réduction et d'oubli formidable qui se met en œuvre à chaque "opération" de connaissance et qui est "inévitable"2. Peut-être est-ce dans cette permanente dérobade du téléspectateur que réside sa faculté à résister?

1 D. Boullier, M. Legrand, F. Gouriou, Je regarde, j'aime, c'est bien: les téléspectateurs jugent la télévision, Paris, Euristic Média, INA, 1990, p. 38.

2 Ibidem. 